Instructions for authors, subscriptions and further details:

http://rise.hipatiapress.com

\title{
El Desencuentro entre Ciencia y Educación; Un Problema Científico-Social
}

Jesús Alberto Valero-Matas ${ }^{1}$

Irene Valero-Oteo ${ }^{2}$

Juan R. Coca ${ }^{1}$

1) Universidad de Valladolid - GIR Tran-REAL Lab

2) Universidad del País Vasco

Date of publication: October $25^{\text {th }}, 2017$

Edition period: October 2017-February 2018

To cite this article: Valero-Matas, J.A., Valero-Oteo, I. \& R-Coca, J. (2017). El Desencuentro entre Ciencia y Educación; Un Problema Científico-Social. International Journal of Sociology of Education, 6(3), 296-322. doi: $10.17583 /$ rise. 2017.2724

To link this article: http://dx.doi.org/10.17583/rise.2017.2724

PLEASE SCROLL DOWN FOR ARTICLE

The terms and conditions of use are related to the Open Journal System and to Creative Commons Attribution License (CC-BY) 


\title{
The Disagreement Between Science and Education: A Scientific-Social Problem
}

\author{
Jesús Alberto Valero-Matas \\ Irene Valero-Oteo \\ Juan R-Coca \\ Universidad de Valladolid \\ Universidad del País Vasco \\ Universidad de Valladolid
}

(Received: 29 April 2017; Accepted: 29 September 2017; Published: 25 October 2017)

\section{Abstract}

This work aims to study the problems to increase technological production system, and the lack of science, technology, engineering and mathematics graduates face of the Western countries. There are many jobs for graduates in experimental sciences in labor market and even so, the Spanish young like in Western countries, prefer social science degrees. Given this fact, we analyze a number of reasons why young students not selected experimental studies of science, even if in the coming years, the main works in the strongest economies will be allocated to these degrees are analyzed. Finally, the paper concludes with some reflections how to act to improve the rates of students in science degrees.

Keywords: school, job, research, science 


\section{El Desencuentro entre Ciencia y Educación; Un Problema Científico-Social}

Jesús Alberto Valero-Matas

Universidad de Valladolid
Irene Valero-Oteo

Universidad del País Vasco
Juan R. Coca

Universidad de Valladolid

(Recibido: 29 Abril 2017; Aceptado: 29 Septiembre 2017; Publicado: 25 Octubre 2017)

\section{Resumen}

Este trabajo pretende abordar la problemática ante la cual se enfrentan los países occidentales ante el incremento tecnológico del sistema productivo, y la carencia de licenciados en ciencias experimentales. En el mercado laboral existen muchos trabajos destinados a licenciados en ciencias experimentales y sin embargo, cada vez, los jóvenes españoles al igual que los jóvenes de otros países occidentales no optan por estudios de ciencias experimentales. Ante esta realidad, este trabajo analiza una serie de causas por las que los jóvenes estudiantes no seleccionan los estudios de ciencias experimentales, aun cuando en los próximos años, los principales trabajos en las economías más potentes estarán destinados a estas titulaciones. El trabajo termina con unas reflexiones como actuar para mejorar los índices de estudiantes en las titulaciones de ciencias.

Palabras clave: escuela, trabajo, investigación, ciencia 
U

no de los grandes problemas económico-educativos de los países occidentales es la escasez de estudiantes de ciencias, tecnología, ingeniería y matemáticas (CTIM- en inglés, STEM). Si se analizan los informes internacionales sobre la situación (Informe Rocard, ROSE, Informe National Science Foundation-USA o la American Association for the Advancement of Science (AAAS)) se observa que existe una profunda fragmentación entre educación científica y mercado laboral. En las sociedades avanzadas cada vez menos jóvenes eligen estudios de STEM y optan por grados en ciencias sociales, jurídicas y humanidades.

El alarmante descenso desde hace décadas de los estudios de ciencia y tecnología, como expone Quagliata (2015) con respecto a los Estados Unidos es que se enfrenta a una crisis de la educación y a la pérdida de su condición de líder en la escena internacional. Los rankings de la juventud americana en la ciencia, ingeniería y matemáticas se han reducido significativamente desde 1970 (National Science Board 2003; Organisation for Economic Cooperation and Development 2006). Ante este hecho, los gobiernos están desarrollando programas de estimulación de la formación científica que solucionen el asunto, pero, aun así, después de décadas intentando animar a los estudiantes a decantarse por estudios de ciencia y tecnología, no se ha logrado evitar la fragmentación. Por el momento, la solución es la incorporación de científicos procedentes de China, India y países de rentas media o baja.

La situación es contradictoria, se necesitan científicos y tecnólogos, el mercado demanda estas especialidades, los jóvenes optan por grados con dificultades en el mercado laboral, los gobiernos no adoptan medidas de contención, y a pesar de todo esto, los jóvenes se ven en la obligación de marcharse, ante la ausencia de proyección laboral, su formación científica no encuentra cabida en el mercado laboral o se ven obligados a desempeñar un puesto de trabajo muy por debajo de su formación, es decir, infra-empleos o supra-cualificación ${ }^{1}$. Con estos mimbres, el panorama educativo, laboral y social se complica o al menos, como diría Beck (2009) alberga una situación de riesgo. ¿Qué se puede hacer? 


\section{Ciencia e Investigación en España}

Desde hace décadas científicos españoles emigran ante la dificultad que tienen para poder hacerse un hueco en el mundo de la investigación en España. Haciendo uso de los datos del INE (2015a) se contempla como un importante número de ingenieros, licenciados en ciencias o científicos han abandonado España en los últimos años y el descenso del número de investigadores a jornada completa. En esta misma línea están las observaciones realizadas por el Banco de España en su boletín económico (2014) y Documento de Trabajo 1503 (2015) donde pone de relieve el peligro de la fuga de cerebros.

De sobra es conocido que cuando un país reduce la inversión en $\mathrm{I}+\mathrm{D}$ dificulta su competitividad, y sobremanera, la capacidad de reindustrialización de emprendimiento necesario para generar valor añadido, y por ende, desarrollo y crecimiento económico. España a diferencia de otros países, como Alemania, Japón, Corea, Israel o USA cuya estrategia fue invertir más en investigación e innovación durante la crisis, redujo el gasto en $\mathrm{I}+\mathrm{D}$. Los grandes avances científicos se han traducido en creación de riqueza para un país, además de ser un instrumento para la mejora de la productividad y la competitividad. Es evidente, como expone el informe de competitividad de la UE de 2013, el del BID o el CES entre otros, la influencia que tiene el gasto en I+D sobre la productividad, con una importante tasa de retorno sobre las inversiones. Por lo tanto, en todo esto se debe valorar que si se desea alcanzar estas cotas de productividad y competitividad en una sociedad bajo un mercado globalizado se ha de tener presente la estrecha relación, de al menos, estas cuatro variables; inversión en $\mathrm{I}+\mathrm{D}$, nivel educativo, producción científica y riqueza.

Según World Economic Forum (2014-2015), España no aparece entre los diez países más competitivos del mundo, fundamentalmente por su baja inversión en investigación. La investigación en España mantiene un sistema cerrado, haciendo uso de la definición de la termodinámica, se habla de un sistema donde no existe intercambio de materia, pero si de energía. Esto es lo que acontece en la investigación española. El sistema cerrado se mantiene principalmente por dos procesos, uno, los centros de investigación y docentes, por norma general, despliegan modelos endogámicos, impidiendo la incorporación de excelentes investigadores o docentes procedente de otros lugares, y dos, en España no cabe (casi) la posibilidad de modelos mixtos de 
investigación y docencia. La inversión de I+D no alcanza los niveles de los principales países más competitivos del mundo, ni se encuentra entre los diez países más inversores de I+D de la EU. (Ver gráfico 1 y tabla I). Por esta razón, resulta complicado, por hacer un símil futbolístico, estar en la Champion League de la investigación.

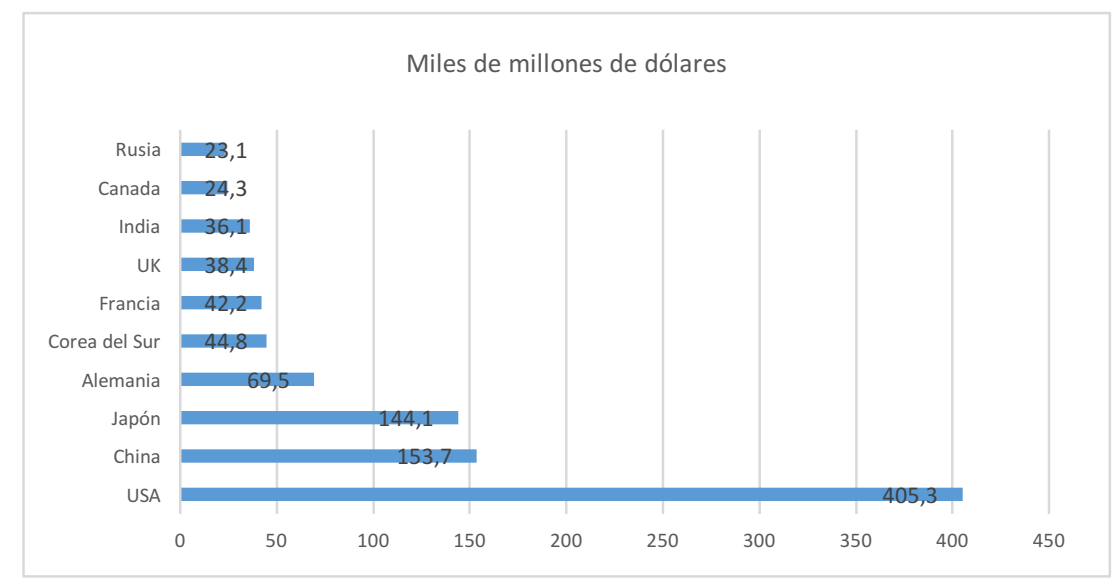

Figura 1. Los diez países que más invierten en I+D en miles de millones de Dólares por (ppp)

Fuente: Elaboración propia a partir del Banco Mundial (2016). 
Tabla 1

Evolución del Gasto de I+D de los 18 países más inversores de la UE y España (PIB Fuente: Elaboración propia a partir datos Eurostat (2016).

\begin{tabular}{|c|c|c|c|c|c|c|c|c|c|c|c|c|c|c|}
\hline & 2002 & 2003 & 2004 & 2005 & 2006 & 2007 & 2008 & 2009 & 2010 & 2011 & 2012 & 2013 & 2014 & 2015 \\
\hline EU & 1,81 & 1,8 & 1,76 & 1,76 & 1,78 & 1,78 & 1,85 & 1,94 & 1,93 & 1,97 & 2,01 & 2,03 & 2,04 & 2,03 \\
\hline Alemania & 2,42 & 2,46 & 2,42 & 2,43 & 2,46 & 2,45 & 2,6 & 2,73 & 2,72 & 2,8 & 2,88 & 2,83 & 2,89 & 2,87 \\
\hline Austria & 2,07 & 2,18 & 2,17 & 2,38 & 2,37 & 2,43 & 2,59 & 2,61 & 2,74 & 2,68 & 2,81 & 2,96 & 3,06 & 3,07 \\
\hline Belgica & 1,89 & 1,83 & 1,81 & 1,78 & 1,81 & 1,84 & 1,92 & 1,97 & 2,05 & 2,15 & 2,24 & 2,42 & 2,46 & 2,45 \\
\hline Dinamarca & 2,44 & 2,51 & 2,42 & 2,39 & 2,4 & 2,51 & 2,78 & 3,07 & 2,94 & 2,97 & 3,03 & 3,08 & 3,02 & 3,03 \\
\hline Eslovenia & 1,44 & 1,25 & 1,37 & 1,41 & 1,53 & 1,42 & 1,63 & 1,82 & 2,06 & 2,43 & 2,58 & 2,6 & 2,39 & 2,21 \\
\hline Espana & 0,96 & 1,02 & 1,04 & 1,1 & 1,17 & 1,23 & 1,32 & 1,35 & 1,35 & 1,32 & 1,27 & 1,24 & 1,24 & 1,22 \\
\hline Estonia & 0,72 & 0,77 & 0,85 & 0,92 & 1,12 & 1,07 & 1,26 & 1,4 & 1,58 & 2,34 & 2,16 & 1,74 & 1,46 & 1,50 \\
\hline Finlandia & 3,26 & 3,3 & 3,31 & 3,33 & 3,34 & 3,35 & 3,55 & 3,75 & 3,73 & 3,64 & 3,43 & 3,3 & 3,17 & 2,90 \\
\hline Francia & 2,17 & 2,11 & 2,09 & 2,04 & 2,05 & 2,02 & 2,06 & 2,21 & 2,18 & 2,19 & 2,23 & 2,24 & 2,26 & 2,23 \\
\hline Hungria & 0,99 & 0,92 & 0,87 & 0,93 & 0,99 & 0,97 & 0,99 & 1,14 & 1,15 & 1,2 & 1,27 & 1,41 & 1,36 & 1,38 \\
\hline Irlanda & 1,06 & 1,13 & 1,18 & 1,2 & 1,21 & 1,24 & 1,39 & 1,63 & 1,62 & 1,53 & 1,58 & 1,58 & 1,51 & 1,52 \\
\hline Italia & 1,08 & 1,06 & 1,05 & 1,05 & 1,09 & 1,13 & 1,16 & 1,22 & 1,22 & 1,21 & 1,26 & 1,3 & 1,38 & 1,33 \\
\hline $\begin{array}{l}\text { Paises } \\
\text { Bajos }\end{array}$ & 1,77 & 1,81 & 1,82 & 1,81 & 1,77 & 1,7 & 1,65 & 1,69 & 1,72 & 1,89 & 1,97 & 1,96 & 2,00 & 2,01 \\
\hline Portugal & 0,72 & 0,7 & 0,73 & 0,76 & 0,95 & 1,12 & 1,45 & 1,58 & 1,53 & 1,46 & 1,37 & 1,33 & 1,29 & 1,28 \\
\hline UK & 1,72 & 1,67 & 1,61 & 1,63 & 1,65 & 1,69 & 1,69 & 1,75 & 1,69 & 1,69 & 1,63 & 1,69 & 1,68 & 1,70 \\
\hline Chequia & 1,1 & 1,15 & 1,15 & 1,17 & 1,23 & 1,31 & 1,24 & 1,3 & 1,34 & 1,56 & 1,79 & 1,91 & 1,97 & 1,95 \\
\hline Suecia & 3,61 & 3,39 & 3,39 & 3,5 & 3,26 & 3,5 & 3,42 & 3,22 & 3,22 & 3,22 & 3,28 & 3,2 & 3,15 & 3,16 \\
\hline Noruega & 1,66 & 1,71 & 1,57 & 1,51 & 1,48 & 1,59 & 1,58 & 1,76 & 1,68 & 1,65 & 1,65 & 1,65 & 1,72 & 1,93 \\
\hline
\end{tabular}

La transferencia de conocimiento de la universidad a la empresa sigue siendo una asignatura pendiente de la sociedad española (Montoro y Mora 2006). La importancia de la colaboración no sólo responde a intereses económicos y promover la innovación empresarial sino también para desarrollar investigación universitaria. De modo que es necesario una estrategia de cooperación para estar en una posición favorable en la economía global del conocimiento. Para ello se deben limar asperezas y cambiar comportamientos. La universidad camina muchas veces ajena a las necesidades empresariales y sociales, y puede aportar mucho con la 


\section{Valero-Matas, Valero-Oteo \& Coca-Ciencia}

investigación básica (Rodriguez y Casani 2007), mientras que las empresas deben eliminar de su imaginario que la universidad está anticuada y no se ajusta a las demandas empresarias (Hidalgo y León 2006).

\section{Currículo Escolar y Ciencia}

Bastantes estudios sostienen que los estudiantes comienzan a perder el interés por la ciencia en los últimos años de primaria o en los primeros cursos de secundaria, y que cuando llegan al bachiller seleccionan itinerarios de ciencias sociales y jurídicas en detrimento de las ciencias experimentales e ingeniería (Murphy y Beggs 2003; Fesham 2004; Vázquez y Manassero 2005; 2008; 2012; Chen y Weko 2009; BØE 2012; Jeampierre-Hallett-Njuguna 2014). Existen otros factores determinantes en la elección de grados diferentes a las ciencias experimentales, tales como la motivación de los docentes, una correcta planificación de los contenidos en ciencias o el currículo de los estudios de secundaria y bachiller.

En el caso español, si uno analiza en profundidad los programas de asignaturas de bachiller comprobará una clara orientación hacia las ciencias sociales y humanas. No se debe tener miedo en decir que España es un país de letras, cuya herencia humanística procede de la primera Universidad: Salamanca. No sólo esto ocurre en el bachiller, también en las asignaturas de la ESO comunes, las ciencias están ausentes, tan sólo aparecen las matemáticas, mientras asignaturas de humanidades o ciencias sociales presentan una amplia oferta. Entre primero y tercero de la ESO, el porcentaje de las asignaturas comunes correspondientes a asignaturas de ciencias es del $33,3 \%$, entretanto HyCS representan el 66,6\%. En las asignaturas optativas, la oferta es, un 25\% de CCExp., y un 75\% de HyCS. En cuarto de la ESO, en las materias comunes, desciende considerablemente el porcentaje de asignaturas de ciencias experimentales hasta el 16\% mientras las asignaturas de $\mathrm{HyCS}$ ascienden al $84 \%$. En bachiller ocurre un tanto de lo mismo, pero prácticamente como exponen varios estudios (Alvarado y Flores-Camacho 2010; Polino 2011) los estudiantes ya en un 89\% han definido sus preferencias por un itinerario u otro en los estudios de secundaria. Por otra parte, como vemos en el caso español, prácticamente con el currículo se orienta a los estudiantes hacia HyCS. 


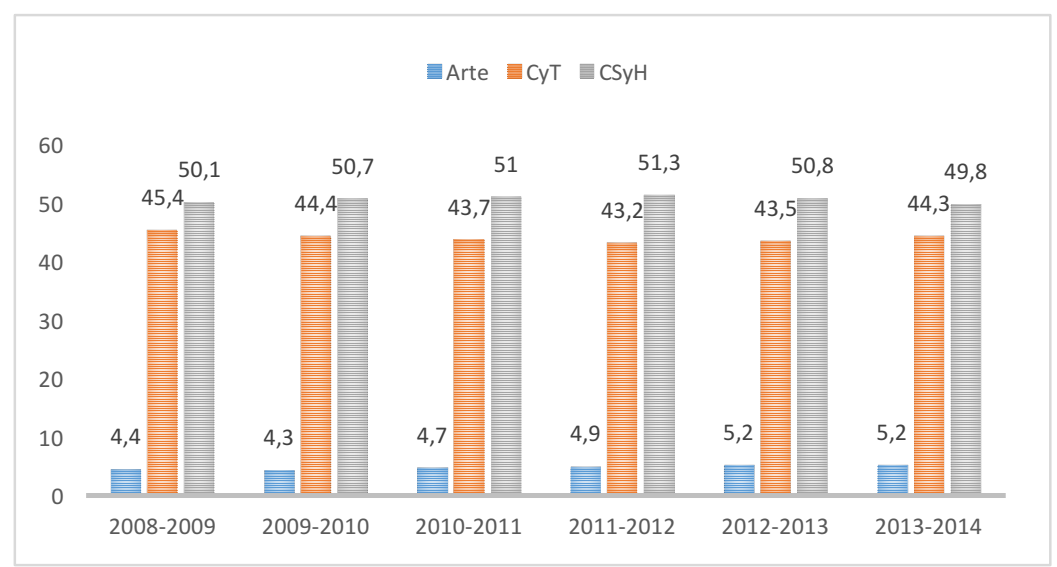

Figura 2. Estudiantes matriculados en Bachiller por ramas de conocimiento Elaboración propia según datos del Ministerio de Educación y Cultura (2016)

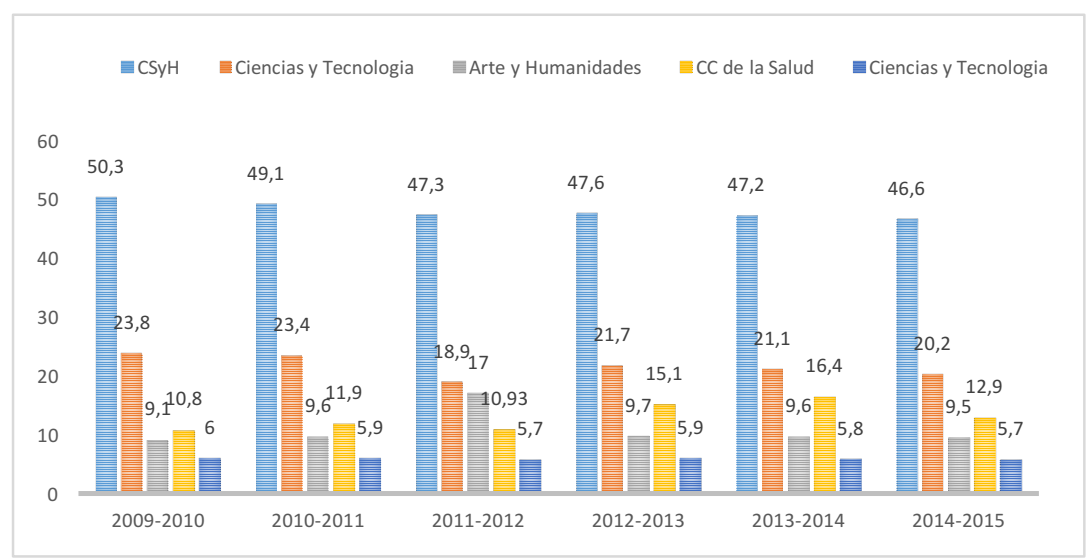

Figura 3. Evolución de la matrícula universitaria por áreas de conocimiento Elaboración propia según datos del MECD (2016) 


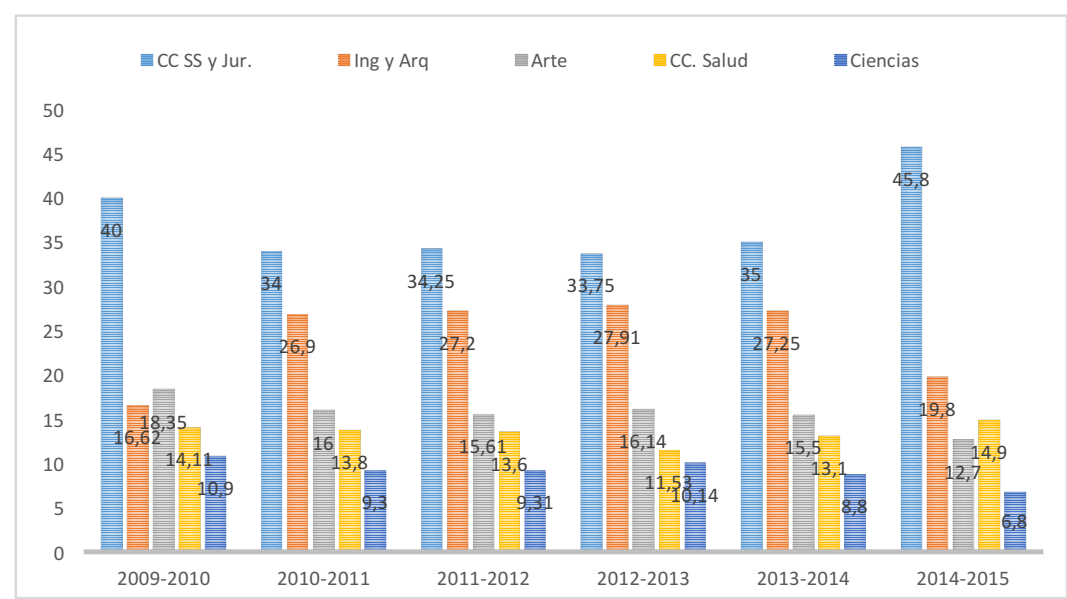

Figura 4. Oferta de titulaciones por áreas de conocimiento Elaboración propia según datos del MECD (2016)

Factores que Influyen en la Elección de Estudios de Ciencias Experimentales o Ciencias Sociales y Humanidades.

La influencia de los padres, por norma general ha sido siempre el principal elemento para decantarse por unos estudios u otros. Si bien, desde hace décadas la libertad individual ha estado bastante presente en los estudiantes, reduciendo el índice de influencia de los padres.

\section{Influencia en la elección de estudios de ciencia}

Los factores que influyen en la elección de una carrera universitaria son muy diversos, y dependiendo de las variables que se estudien, estos coincidirán o no. Analizando nuestro estudio (2014, longitudinal de cinco años de duración) y combinándolo con el análisis de otros estudios nacionales e internacionales se desprende que la motivación, los medios de comunicación e internet y la familia aparecen como los aspectos más destacados en la elección de una carrera universitaria. En el caso español se adiciona un aspecto sobremanera importante y fundamental, poseer un título universitario ${ }^{2}$.

En el caso de los estudios realizados por Vázquez-Alonso y ManasseroMas $(2008 ; 2015)$ los padres no ejercen demasiada influencia a la hora de la elección de grados en ciencias experimentales, pero si son determinantes los 
buenos profesores y los tutores, los temas científicos y, en menor medida los libros y la divulgación científica. En el estudio de Everis (2012) los padres aparecen como el primer activo en la elección de una carrera universitaria, en segundo lugar, aparecen las consultas a internet de temas científicos, videos, etc., y en tercer lugar, los profesores implicados, los tutores, hermanos y amigos. En un estudio previo a estos, llevado a cabo por Porto y Mosteiro (2000) sobresalían dos factores sobre los demás: me gusta mucho y tengo posibilidades de encontrar trabajo. En los trabajos de BØE (2012) y Eccles and Wigfield (2002) los elementos más influyentes recaen en la percepción personal hacia los estudios y el mercado laboral. Tiempo atrás los estudiantes españoles valoraban como principales causas en la elección de un título universitario, la percepción personal hacia los estudios, el mercado laboral y por supuesto, poseer un título universitario. Con lo cual, dos generaciones después los factores selectivos han mudado.

\section{Educación primaria y secundaria}

Como ponen de relieve diversos estudios y artículos, los estudiantes de primaria ven la ciencia como algo curioso, los motiva y realizan constantes preguntas sobre asuntos científicos. ¿Por qué giran alrededor del sol los planetas? ¿Cómo se producen las olas? Como señala Rundell (2013) los estudiantes plantean cuestiones interesantes a los docentes sobre el genoma humano, las pilas de hidrógeno, el cambio climático, lo que hace que los docentes ante dichas cuestiones desplieguen soluciones creativas, y en ocasiones los ponen en situaciones comprometidas para dar respuestas a dichas preguntas. Es una parte más del desarrollo del ser humano y su proceso cultural. La cuestión se invierte a medida que los estudiantes avanzan en el nivel escolar perdiendo esa curiosidad, las asignaturas de ciencias se hacen complicadas, difíciles y cuando terminan la secundaria hay menos estudiantes en los itinerarios de ciencias experimentales que en otros itinerarios. Además, en los primeros años de la universidad, se observa cómo se produce un transvase de estudiantes de estudios de ciencias a estudios de ciencias experimentales e ingeniería a estudios de $\mathrm{CSyH}^{3}$.

En este orden de cosas, es necesario plantearse qué factores intervienen en el rechazo a las ciencias experimentales a medida que se avanza en la formación, es decir, desde primaria hasta la universidad. Desde esta perspectiva es necesario estudiar diferentes cuestiones, que intervienen 
directamente en la orientación académica, como son los diseños de los programas académicos, que se encuentran especialmente centrados en las ciencias sociales (Pérez de Landazabal, Caldeira y Otero, 2011). Importante a la hora de configurar el perfil académico profesional del joven. En segundo lugar, el desasosiego de los estudiantes con las asignaturas de ciencias, pues según estos, son demasiado aburridas, resulta desagradables estudiarlas, la ciencia a diferencia de lo que se piensa no abre la curiosidad a los estudiantes, no perciben la ciencia como algo positivo para ellos y tampoco la ven como proyección de futuro (ver tabla 2, resultados del estudio). En tercer lugar, no existe una línea de motivación científica en las escuelas, en otras palabras, la utilización de instrumentos, experiencias y contenidos científicos que atraigan a los estudiantes hacia las ciencias. En nuestro estudio, se ha detectado como los estudiantes demandan más prácticas, trabajo participativo y un contacto directo con las ciencias, es decir, más laboratorios, realidades enunciadas en el trabajo de Korpershock, Kuyper y Wert (2015), en otras palabras, la necesidad de modelizar a los estudiantes hacia los estudios de la ciencia (Ellington 2006; Chang et.al., 2008). En cuarto lugar, la ciencia que se enseña en primaria suele ser bastante complicada incluso para los propios docentes, lo que indudablemente genera un rechazo en los estudiantes. En estos términos lo percibían Murphy y Beggs (2003) cuando señalaban:

En ciencias naturales, a los niños de educación primaria se les familiarizó con plantas y animales, haciendo uso de ejemplos desafiantes que estimularan su interés y curiosidad, en contraposición de recurrir a nombrar de manera engorrosa los nombres de las flores y plantas y partes del cuerpo humano, tales como 'ovulo o el omoplato. Por lo tanto, pensamos que a los niños de primaria sus profesores no les deben enseñar aspectos de la ciencia que son demasiado difíciles incluso para sus profesores (2003, p.114).

A pesar de ello, se siguen manteniendo estos modelos de enseñanza basados en el aprendizaje memorístico, lo que viene a ser aprender para olvidar. Esto ocurre porque se ha abandonado la enseñanza basada en adiestramiento de razonamientos, exposición de experiencias, creación de conocimiento, resolución de problemas e instrucción en el método científico, en favor de un entrenamiento memorístico y casuístico. Todo lo contrario de 
lo requerido en ciencias experimentales. En quinto lugar, los estudiantes perciben la falta de motivación de los docentes en todos los niveles educativos, la ausencia de interés del profesorado por enseñar ciencias y tecnología (Strayhom, 2010). Se puede enumerar un elenco de razones que muestran esa displicencia, como la pérdida de implicación y responsabilidad de la academia con los estudiantes y la sociedad, pasando a tornarse como un empleo más. La carencia de formación científica en los docentes de primaria impide la exposición y enseñanza de los avances científicos, como indican los estudios de Appleton (2013) y Coll y Earnes (2008). Según dichos estudios los estudiantes del grado de educación piensan que a sus profesores no les gustaba la enseñanza de las ciencias, especialmente por su escaso conocimiento en la materia: "los profesores de primaria no tenían suficiente formación en contenido científico, y además presentaban actitudes negativas hacia la ciencia" (Appleton, 2013, p. 119), en el caso de Coll y Earner (2008), los estudiantes de grado en educación revelaban que a ellos tampoco les gustaba la ciencia, puesto que "resulta muy abstracta y por lo tanto complica su comprensión. Además, los profesores lo hacen complicado, y se realizan pocas prácticas, dificultando el aprendizaje puesto que como más se aprende es haciéndolo con las manos" (Coll y Earner, 2008, p. 98). Un comportamiento similar acontece en España, como se observa en la tabla 2, los datos de los estudiantes que se encuentran incómodos con la ciencia son mucho más altos en los estudiantes del grado en educación que el resto.

Con una percepción de este orden resulta complicado enseñar ciencia a los bisoños. En esta misma línea, como han expresado un alto porcentaje de estudiantes de educación (tabla 2), las ciencias no les aportan gran cosa, y además un $62 \%$ considera que la ciencia tiene más efectos negativos que positivos. La ciencia debería poder solucionar los grandes problemas sociales del mundo, como el hambre, la sequía y demás, pero no interesa a las grandes empresas. Es cierto que en el caso de la ciencia médica la sensación es muy diferente, un $88,2 \%$ la ve como fundamental en la mejora de la calidad de vida de las personas y el desarrollo social. Con ideas de esta clase resulta arduo una enseñanza positiva de las ciencias experimentales que se transmita en un incremento de científicos y tecnólogos. 


\section{Valero-Matas, Valero-Oteo \& Coca-Ciencia}

\section{Tabla 2}

Ciencia, educación y mercado laboral

\begin{tabular}{|c|c|c|c|c|}
\hline & $\begin{array}{r}\text { Estudiantes de } \\
\text { Itinerarios de } \\
\text { Ciencias } \\
\text { Experimentales } \\
\end{array}$ & $\begin{array}{r}\text { Estudiantes de } \\
\text { otros Itinerarios }\end{array}$ & Sig. & $\begin{array}{l}\text { Test de } \\
\text { Levene }\end{array}$ \\
\hline $\begin{array}{l}\text { Las clases son demasiado teóricas y me } \\
\text { aburre }\end{array}$ & 3,39 & 3,06 & ,006 & ,338 \\
\hline $\begin{array}{l}\text { El estudio de las Ciencias } \\
\text { Experimentales me resulta algo pesado } \\
\text { porque no veo su utilidad }\end{array}$ & 2,11 & 2,89 &, 000 & ,989 \\
\hline $\begin{array}{l}\text { Me gusta resolver problemas } \\
\text { relacionados con las Ciencias } \\
\text { Experimentales }\end{array}$ & 3,52 & 2,28 &, 000 & ,076 \\
\hline $\begin{array}{l}\text { Me interesa el estudio de las Ciencias } \\
\text { Experimentales porque los considero } \\
\text { importante como preparación para } \\
\text { encontrar un puesto de trabajo }\end{array}$ & 3,59 & 2,58 &, 000 &, 015 \\
\hline $\begin{array}{l}\text { El estudio de las Ciencias } \\
\text { Experimentales me produce satisfacción }\end{array}$ & 3,45 & 2,35 &, 000 &, 011 \\
\hline $\begin{array}{l}\text { Me resulta desagradable estudiar las } \\
\text { asignaturas de Ciencias Experimentales }\end{array}$ & 2,04 & 3,07 &, 000 &, 007 \\
\hline $\begin{array}{l}\text { El profesorado tiene poca formación en } \\
\text { las Ciencias Experimentales } \\
\text { (desconocimiento) }\end{array}$ & 2,40 & 2,74 & 004 & ,311 \\
\hline $\begin{array}{l}\text { Una de las causas de mi desinterés por } \\
\text { la Ciencia está en el profesor }\end{array}$ & 2,61 & 2,89 &, 043 &, 436 \\
\hline $\begin{array}{l}\text { El profesorado no muestra interés en } \\
\text { enseñar Ciencias y tecnología }\end{array}$ & 2,76 & 2,46 &, 009 & ,273 \\
\hline $\begin{array}{l}\text { Las Ciencias Sociales y Humanas tienen } \\
\text { más aplicación en la vida social }\end{array}$ & 2,57 & 3,84 &, 000 & 000 \\
\hline
\end{tabular}

Aunque ha habido un incremento en la alfabetización científica (FECYT, 2014), el porcentaje de jóvenes decantados hacia los estudios de ciencias y tecnología continúan siendo bajos. Una sociedad cuyos porcentajes de estudiantes de áreas de ciencias y tecnología son bajos, están poniendo en riesgo su propio sistema productivo, National Science Foundation (2014) apunta que, en los Estados Unidos en el año 2020, nueve de cada diez ocupaciones estarán relacionados con las ciencias, fundamentalmente matemáticas, ingeniería y salud. La EU prevé para el año 2020, los países de la EU (2014) necesitaran unos 700.000 científicos y tecnólogos para cumplir los objetivos marcados por Horizon 2020. En estos momentos, a excepción de Finlandia, Noruega y Dinamarca, el resto están muy alejados de cumplir dichos objetivos. Y por lo tanto se deberá recurrir a contratar esta fuerza de 
trabajo de países de ingresos medios o bajos.

El crecimiento de científicos no americanos en los Estados Unidos ${ }^{4}$ ha sido de 1.827.000 en diez años, y como señala la National Science Foundation (2014) existe cierta preocupación por los estudios de ciencia, que siguen estancados en las universidades americanas, lo que obliga a incorporar científicos procedentes de otras partes del mundo. En estos mismos datos, se observa como 851,000 científicos europeos han decidido moverse a este país. Con lo cual, Europa se descapitaliza de sus propios científicos incrementando aún más la problemática científica.

Estados Unidos lleva décadas planteándose y buscando programas de estimulación científica en la educación elementary, middle y High school como el proyecto 2061 desarrollando por la Asociación Americana para el Avance de la Ciencia (AAAS) desde 1985, (AAAS, 1989; Van Zee, et al., 2005; Porter, et al., 2006; Kali \& Linn 2008; Lloyd, 2009; Rundell 2013). Las acciones de fomento de la ciencia y la tecnología son incesantes en los USA y en el año 2006, ante la escasez o necesidad de científicos y tecnólogos, el Congreso de los Estados Unidos anunció el Programa American Competitiviness Iniciative Act, cuyo objetivo es hacer a los Estados Unidos más competitivo a través de la investigación, el desarrollo y la educación. A pesar de estos programas, incentivos y mejora de la docencia y docentes en la educación, el país continúa sin alcanzar los objetivos marcados. Como apuntan algunos estudios, resulta mucho más rentable y práctico a los estudiantes ser economista o abogado, ya que estas profesiones generan mayores beneficios, y es más fácil alcanzar el éxito (Long, 2013). Desde 2014 parece haber habido un cambio en los universitarios, pues un estudio realizado por el profesor Lazowska de la Universidad de Washington, ha detectado un incremento sustancial de estudiantes de computación en varias universidades americanas.

Empero teniendo un problema común, existen grandes diferencias entre España y EE.UU. En EE.UU las escuelas despliegan un amplio abanico de medidas a favor de las ciencias experimentales, (instrumentos pedagógicos, programas de fomento la ciencia, visitas a centros especializados de ciencia, huertos científicos, etc.), mientras España sigue con los mismos programas educativos de hace cincuenta años y sin adaptarse a los cambios. Las dificultades para desarrollar una carrera científico académica reside en las condiciones poco favorables de la academia, pero no para encontrar empleo 


\section{Valero-Matas, Valero-Oteo \& Coca-Ciencia}

en empresas científico-tecnológicas, como apuntan especialistas en el mercado laboral ${ }^{5}$. En cambio, son puestos aceptados por científicos de otros países, como India, China o España entre otros. Mientras en el caso español, la dificultad es diversa, puesto que no se generan plazas de científicos titulares en los centros de investigación, la vida académica en las universidades se ha complicado, y existe una escasez de empresas científico tecnológicas que puedan absorber los graduados en ciencias. Con este panorama, resulta fácil entender la fuga de científicos y tecnólogos, así como la escasa proyección hacia estas titulaciones. Aun mientras el mercado demanda profesionales con titulación en ciencia e ingeniería. Los jóvenes no lo perciben así, sino que, las ciencias sociales tienen más salidas profesionales que las ciencias experimentales (ver tabla 2).

\section{Ocupación y educación científica}

Si analizamos los datos de diferentes medios, INE (2015b), MECD (2016) o Adeco (2015) se observa que no coinciden las titulaciones más demandadas en el mercado laboral español. Sin embargo, aparece un nexo de unión, las titulaciones de ciencias presentan mayores posibilidades laborales. Según la encuesta del INE (2015b) las titulaciones con mayor empleabilidad (ver gráfico 5) corresponden a ciencias e ingeniería, con excepción de Investigación y técnicas de mercado. En el aspecto contrario, en las titulaciones con mayor desempleo tan sólo se encuentra una titulación de ciencias, diplomado en navegación marítima. Centrándose en la valoración de Randstad, las titulaciones más demandadas por el mercado laboral son administración y dirección de empresas (ADE), química e ingenieros industriales especializados en la rama de la mecánica. Otras con menor medida, pero también con alta ocupación están Económicas, ingeniería técnica de informática, derecho, aeronáutica, ingeniería electrónica, estas con alta movilidad en el extranjero y farmacia. 


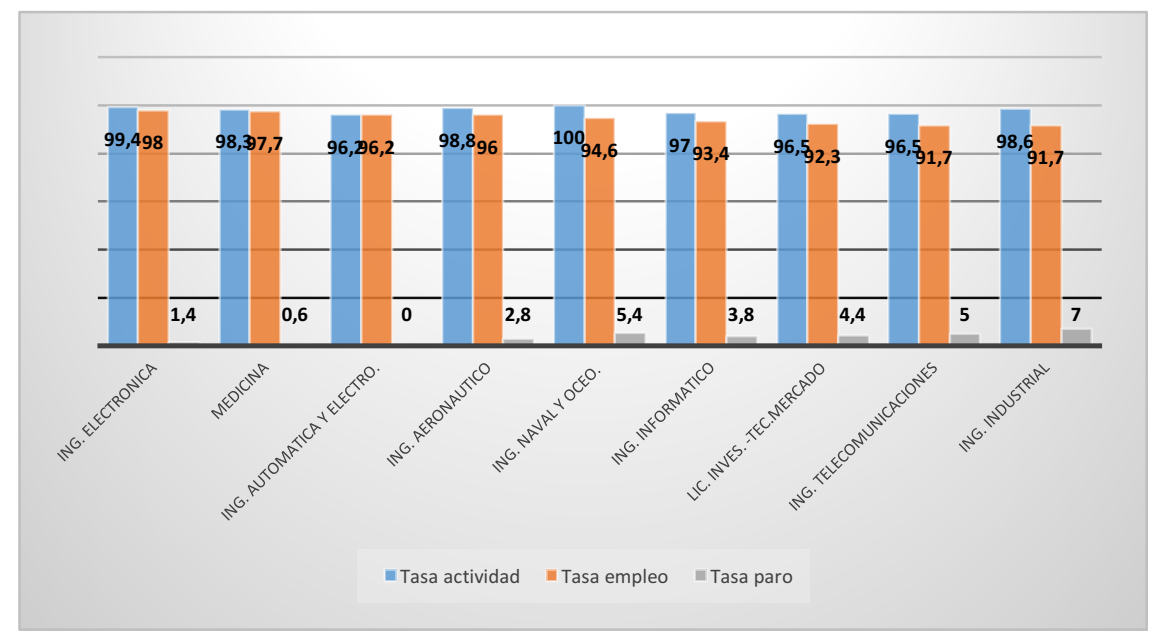

Figura 5. Titulaciones con mayor tasa de empleo Elaboración propia según datos del INE (2015b)

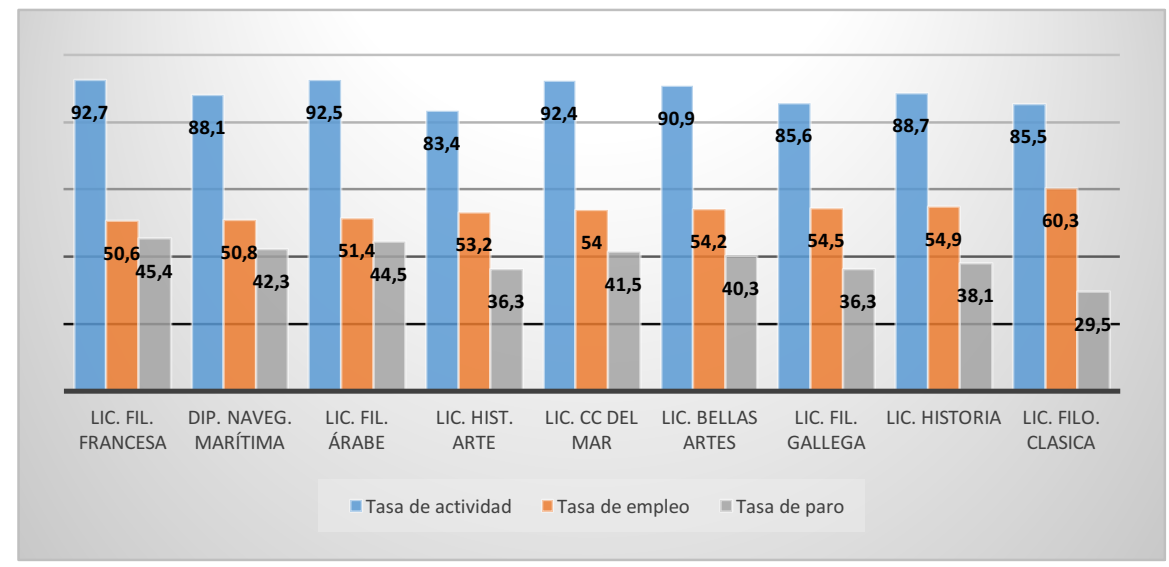

Figura 6. Titulaciones con menos tasa de empleo Elaboración propia según datos del INE (2015b) 


\section{Valero-Matas, Valero-Oteo \& Coca-Ciencia}

En el estudio de Adeco (2015) sitúa en el primer lugar a ADE, seguido de ingeniería informática, y hallándose tres titulaciones de ciencias sociales y jurídicas economía, marketing y Relaciones laborales entre las diez más demandadas.

El análisis del Ministerio de Educación tampoco coincide demasiado con el resto, pues tan sólo ciencias actuariales y financieras están en la liga de las diez titulaciones más demandadas, el resto se corresponden con ingenierías, ciencias de la salud y ciencias. En las titulaciones universitarias menos demanda, están dos ingenierías, y el resto son titulaciones de humanidades.

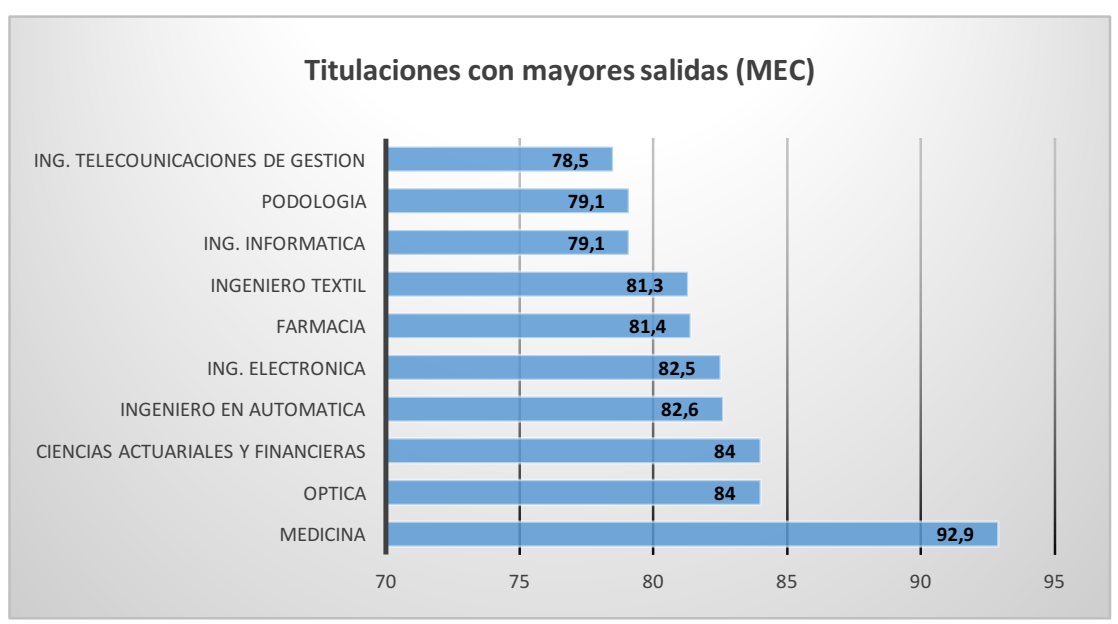

Figura 7. Titulaciones con mayores salidas

Elaboración propia a partir de datos del MECD (2016) 


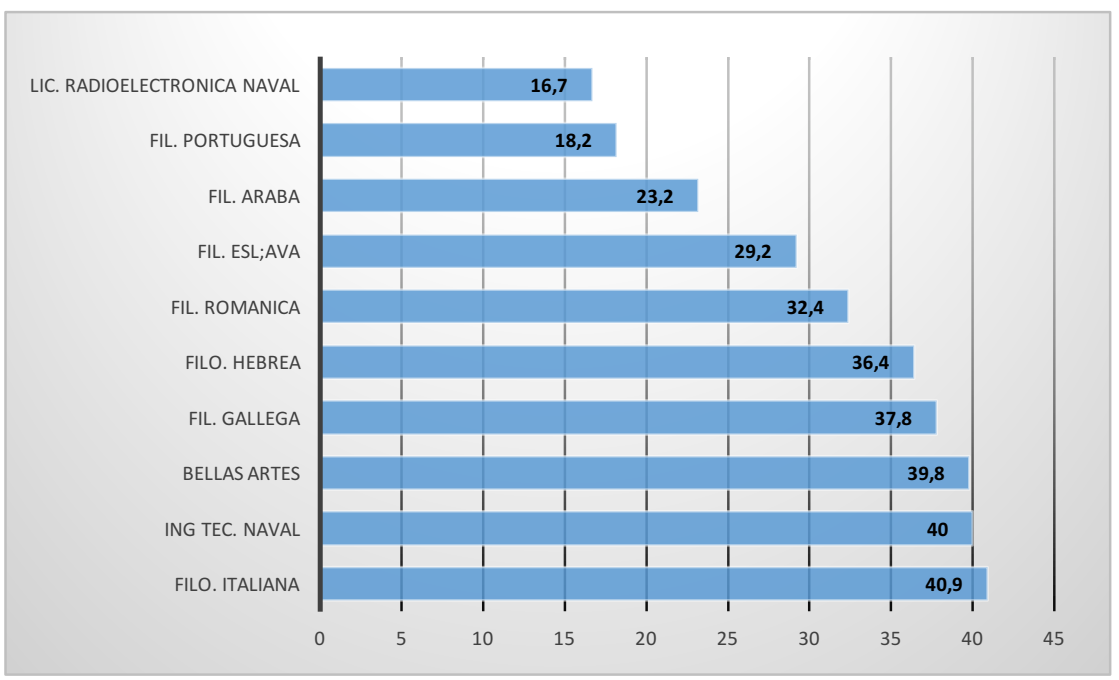

Figura 8. Titulaciones universitarias menos demandadas Elaboración propia a partir de MECD (2016)

Ateniéndose a las observaciones de la EU con acuerdo al Reto 2020 de una Europa en un mundo cambiante, inclusiva, innovadora y reflexiva, pone de relieve la necesidad de profesionales en áreas científico tecnológicas. Tanto el European Centre for the Development of Vocational Training ${ }^{6}$ en sus análisis sobre las necesidades copentenciales de los profesionales como el incremento de profesionales altamente cualificados en STEM (Science, Technology, Engineering \& Mathematics), se verá incrementado entre un $14 \%$ y $16 \%$ en los últimos cinco años. En esta misma línea va el informe mundial de Randstand employer branding 2015, que la demanda laboral de profesionales STEM (Science, Technology, Engineering \& Mathematics) crecerá por encima de la del resto de titulaciones. Solo un $1 \%$ de los universitarios españoles responde a este perfil. Lo cual, la situación está bastante complicada para cubrir, siempre bajo estos estudios, la demanda empresarial. En los Estados Unidos se enfrentará a una situación similar, pues instituciones como la Science National Foundation (2014) y Brookings Institution (2014) prevén que en los Estados Unidos para el año 2020 se necesitaran cubrir 20 millones de nuevos empleos, y cuyos perfiles laborales responderán a las STEM, como muestra el índice de las diez áreas donde se contratará más personal, 1. Extracción y análisis de datos (los big data), 2. 


\section{Valero-Matas, Valero-Oteo \& Coca-Ciencia}

asesoramiento y terapia en salud mental,3. Ingeniería y tecnología de la computación, 4. Investigación en ingeniería, matemáticas, química, biología, biotecnología y otras ciencias. 5. Veterinaria, 6. Ciencias de la salud humana (medicina, enfermería, fisioterapia, etc.) 7. Ciencias medioambientales. 8. Mercado de Finanzas e inversiones, 9. Gestión y administración de empresas y 10. el mercado de la pequeña y mediana empresa.

Ante este panorama, cabe plantearse varias cuestiones, ¿Por qué si en el mercado laboral demanda titulaciones de ciencias y tecnología, los estudiantes se decantan por titulaciones de CCSSyH? ¿Por qué los estudiantes prefieren carreras de CCSSyH, si tienen más tasas de desempleo? ¿Por qué el Ministerio de Educación y las Consejerías sabiendo que las tasas de empleo son mayores en las titulaciones de ciencias y tecnología, siguen ofertando más plazas de CCSSyH? ¿Por qué no se interviene más en los planes de estudio y en los programas de formación en educación primaria y secundaria?

\section{Valoraciones Finales}

La situación para los próximos años se torna harto complicada para España, sino se adoptan medidas educativas y por supuesto económicas que lleven a cubrir los puestos de trabajo demandados por el mercado en materia de ciencia y tecnología. Este asunto no es nuevo, sino que el descenso se viene produciendo desde la década de los 60 de la pasada centuria. La pregunta es ¿Por qué no se han puesto medidas con anterioridad? Sencillamente porque la situación se iba solventando y los jóvenes en un sitio o en otro, encontraban trabajo. En estos momentos, según dicen los datos, la empleabilidad será más complicada porque se requerirá mayor especialización, cómo indica European Centre for the Development of Vocational Training se crearan en los próximos años en España, casi dos millones de empleos, están destinados a perfiles STEM. Como apunta Suchman (2014, p. 657) nuestra economía necesita de una fuerza de trabajo de alta formación educativa en STEM, pero las proyecciones de la misma que están siendo muy debatidas, indica claramente que las necesidades de la fuerza de trabajo están muy por encima del suministro humano. En los grados de matemáticas, la presencia de estudiantes es casi testimonial en las universidades españolas. Ante este horizonte, posiblemente los titulados en CSyH desempeñen trabajos muy alejados de su formación. 
La importancia del fomento y del cambio de modelos de enseñanza en las escuelas es fundamental si se desea que los estudiantes se orienten a estos estudios científicos y tecnológicos. Programas de aprendizaje activo, ciencia interactiva, juegos científicos de capacitación reflexiva acorde a las edades, y un sin fin de técnicas que ayuden a interactuar al estudiante, al docente y a la ciencia. Que los docentes encargados de programas y asignaturas de ciencias, presenten la titulación correspondiente o al menos tengan una formación científica, porque de lo contrario difícilmente podrá enseñar ciencia. Por último, en este sentido, es muy importante la motivación y la formación de los docentes, porque si no se entenderá como un puesto de trabajo más, y la educación es más que eso.

Es necesario superar la brecha entre las ciencias experimentales y las ciencias sociales y humanas, y buscar alternativas a este desequilibrio, y abandonar el mensaje manido de la brecha entre ricos y pobres. Porque en los países de rentas medias o bajas, las personas con pocos recursos y que estudian en la universidad focalizan sus estudios en ciencia y tecnología, mientras que aquellos con capital, optan por titulaciones jurídico financieras (Banco Mundial 2014; UNESCO, 2014).

Se debe continuar reforzando la imagen de la ciencia en la sociedad. Los esfuerzos de diferentes organismos e instituciones públicas y privadas han logrado que la ciencia tenga una mejor percepción en la sociedad (FECYT, 2014), pero es insuficiente porque no se ha transmitido en un incremento de científicos y tecnólogos en las universidades españolas. Probablemente como apunta Elias (2008) la divulgación científica está en auge, pero algo se está haciendo mal porque no se ven resultados. Será necesario analizar la divulgación científica y sus resultados, a modo de buscar cómo crear una mayor implicación de los jóvenes con la ciencia. Como expone Quagliata (2015) diversas universidades en los Estados Unidos promueven The University Festival Promotes STEM Education, con el objeto de atraer estudiantes a los estudios STEM. Desde que se celebra esto ha habido un aumento de jóvenes en estos estudios. Es más, en el caso concreto de su universidad, antes del pre-evento era del 40,5\% los estudiantes decantados por los estudios de ciencias, y después del evento, ascendió a un 46,4\%. Por lo tanto, este tipo de actividades pueden ser una buena atracción a los estudios STEM.

Resulta necesario un cambio social educativo importante, pues no se puede 


\section{Valero-Matas, Valero-Oteo \& Coca-Ciencia}

exclusivamente reorientar a la población estudiantil al mundo de la Universidad cuando existe, parafraseando a Popper (2000), un tercer mundo, la Formación Profesional (FP) quien presenta altos índices de colocación en módulos de ciencia y tecnología (MECD, 2015). Como bien señala la OCDE (2012) España presenta una de las tasas más altas de titulados universitarios, incluso por encima del gran referente, Finlandia, pero también de universitarios en trabajos con una cualificación inferior a su titulación (MECD, 2015). Y una de las ratios menores en FP (OCDE, 2012). Todavía está presente en el imaginario de la ciudadanía ${ }^{7}$, la expresión "el que vale para bachiller y el que no para FP". Mientras esto esté vivo en la sociedad, modificar comportamientos será complejo.

La sociedad se fija mucho en los resultados de los informes Pisa de otros países, especialmente Finlandia. Porque no realizar una introspección interna y ver los resultados de Castilla y León o Navarra, donde los discentes están por encima del promedio de la OCDE en Matemáticas, Ciencias y Lectura (OCDE, 2012) y además, los estudiantes de estas Comunidades Autónomas se decantan más por bachilleratos de ciencias y tecnología que por otros (MECD, 2015). Por lo tanto, quizá debemos abandonar esa teoría "lo de fuera es mejor que lo de dentro", y se analice lo que funciona bien dentro, y se tome de fuera lo que no funciona bien dentro.

Uno de los principios básicos de la libertad se fundamenta en que el sujeto debe decidir por sí mismo, y, por ende, no se puede solicitar a los estudiantes que estudien una carrera porque tiene muchas salidas profesionales, si ellos prefieren una titulación de CSyH. El 92\% de los estudiantes consultados en nuestro estudio y matriculados en grados de CCSSy H creen que existen más salidas profesionales en ciencias sociales y humanidades que en las ciencias experimentales. Por consiguiente, si en el imaginario de las personas se tiene una percepción, y por mucho que diga el mercado, la familia y demás factores, los estudiantes seleccionaran esa titulación. Si se desea modificar comportamientos, una de las cuestiones donde se debe incidir es en mostrar la realidad. La tarea de las administraciones, si quieren modificar comportamientos por el bien de las personas y de la sociedad, es implementar medidas efectivas de incentivación y motivación por las ciencias experimentales.

Por último, introducir en la educación un valor indispensable en las sociedades globalizadas en constante cambio, la creatividad (Valero-Matas, 
et. al., 2016). La creatividad científica ayuda a resolver problemas, redefinir situaciones, fomentar la agudeza humana, desplegar la originalidad, en definitiva, dotar al sujeto de diferentes mecanismos para hacer frente a esta sociedad en constante transformación. La creatividad se adquiere, y en consecuencia se precisa utilizar procesos de aprendizaje creativos, basados en la imaginación. Con esto se trata de mostrar que tanto en la educación inicial, se necesita dotar a los escolares de instrumentos imaginativos que desarrollen esa creatividad, y que se proyecte a lo largo de todo el proceso educativo, es decir hasta completar sus estudios universitarios.

\section{Notas}

${ }^{1}$ Ver informe de la Fundación conocimiento y desarrollo, 2014.

${ }^{2}$ Esto sin ninguna duda para las personas es un valor importante, puesto que indica formación. Pero también es cierto que no todas las titulaciones existentes en el mercado tienen ubicación laboral en sí mismas. Hecho que genera cierta frustración y sobreformación en puestos de trabajo. Por ello, se hace necesario explicar que un título no es sinónimo de empleo.

${ }^{3}$ Ver MEC datos y cifras del sistema universitario español diversos años, viene a ser entre un 17,5\% y 19,8\% (el cálculo es nuestro), independientemente de aquellos que abandonan los estudios de Ciencias experimentales e ingeniería.

${ }^{4}$ Ver Birthplace of immigrant and US citizen Scientist and engineers: 2003-2013

${ }^{5}$ Ver http://www.scientificamerican.com/article/does-the-us-produce-too-m/

${ }^{6} \mathrm{http}$ ://www.cedefop.europa.eu

${ }^{7}$ Entre otras cuestiones porque no se ha incidido en modificar dicha percepción, pues a los políticos, responsables de las políticas educativas de España los ha venido muy bien. Las consecuencias de esto, las lleva sufriendo la sociedad desde décadas, que repercuten directamente sobre el sistema productivo.

\section{Referencias}

Adecco (2015) Informe Infoempleo Adecco. Oferta y demanda de empleo en España 2014. Madrid; Adecco.

Alvarado Rodríguez, M-E. \& Flores Camacho. F. (2010). Percepciones y supuestos sobre la enseñanza de la ciencia. Las concepciones de los investigadores universitarios. Perfiles Educativos, XXXII (128), 1026.

American Association for the Advancement of Science. (1989). Science for all Americans: A Project 2061 report on literacy goals in science, 
mathematics, and technology. Washington, DC: American

Association for the Advancement of Science.

Appleton, K. ed. (2013). Elementary Science Teacher Education. New

York: Routledge.

Asghar, A. R., Ellington, R., Rice, E., Johnson, F., \& Prime, G. M. (2012).

Supporting STEM Education in Secondary Science Contexts.

Interdisciplinary Journal of Problem-based Learning, 6(2), 23-48.

doi: 10.7771/1541-5015.1349

Banco de España. (2014). Boletin Económico, 9/14. Madrid. Banco de

España.

Banco Mundial. (2016). Informe sobre el desarrollo mundial 2016. Banco

Mundial: Washington DC.

Banderly, B. L. (2010). Does the U.S. Produce Too Many Scientists?

American Scientific, February, 22, 1-21.

Beck, U. (2009). La sociedad del riesgo global. Madrid: Siglo XXI.

Bøe, M. V. (2012). Science choices in Norwegian upper secondary school:

What matters? Science Education, 96(1), 1-20.

Brooker, CH. (2009). The real Global Warming Disaster. London:

Continuum.

Brookings Institution (2014) Still Searching: Job Vacancies and STEM

Skills. Washington DC: Brookings Institution.

CES. (2015). Informe la competitividad de la empresa española en el mercado interno: estructura productiva, importaciones y ciclo económico. Colección informes 01/2015. Madrid: CES.

Chang, M. J., Cerna, J. Han, J., \& Saenz. V. (2008). The contradictory roles of institutional status in retaining underrepresented students in biomedical and behavioral science majors, The Review of Higher Education, 31(4), 433-464.

Chen, X., \& Weko. T. (2009). Students who study Science, Technology, Engineering, and Mathematics (STEM) in postsecondary education. Washington DC: US Department of Education, NCES.

Chung, C.J. Cartwright, C \& Cole. M. (2014). Assessing the impact of an autonomous robotics competition for STEM education, Journal of STEM Education: Innovations and Research, 15, 24-34.

Coll, R.K., \& Earnes. C. (2008). Developing and understanding of Higer education science and enginnering learning communities, Research 
in Science and Technological education, 26 (3), 245-257. doi:

$10.1080 / 02635140802276413$

Comisión Europea/EACEA/Eurydice. (2012). El desarrollo de las

competencias clave en el contexto escolar en Europa: desafíos y oportunidades para la política en la materia. Informe de Eurydice. Luxemburgo: Oficina de Publicaciones de la Unión Europea.

Eccles. J.S., \& Wigfield. A. (2002). Motivational Beliefs, Values and Goals. Annual Review Psychology, 52, 109-132. doi:

10.1146/annurev.psych.53.100901.135153

Elias, C. (2008). La razón estrangulada, Barcelona, Debate.

Ellington, R.M. (2006). Having their say: Eight high-achieving African-

American undergraduate mathematics majors discuss their success and persistence in mathematics. Unpublished doctoral dissertation, University of Maryland: College Park.

European Union (2014) Eurostat. Science, Technology and Innovation.

Bruselas: EU.

Everis (2012) Factores influyentes en la elección de los estudios científicos, tecnológicos y matemáticos Visión de los estudiantes de $3^{\circ}$ y $4^{\circ}$ de ESO y Bachillerato. Madrid: Everis Group

FECYT. (2014). VII Encuesta de percepción social de la ciencia. Madrid: FECYT.

Fensham, P.J. (2004). Real World Contexts in PISA Science: Implications for Context- Based Science Education, Journal of Research in Science Teaching, 46(8), 884-896. doi: 10.1002/tea.20334

Fundación Conocimiento y Desarrollo. (2014). Informe CYD 2014. Artículo en línea. Recuperado de: http:/www.fundacioncyd.org/informecyd/informe-cyd-2014.

Hidalgo, A., \& León. G. (2006). La importancia del conocimiento científico y tecnológico en el proceso innovador, RIGIT, 38, 1-17.

INE (2015a) Estadistica de variaciones residenciales. Madrid: INE.

INE (2015b) Encuesta sobre inserción laboral de titulados universitarios.

Madrid: INE

Izquierdo. M.J. Jimeno, F. \& Lacuesta. A. (2015). Spain: from immigration to emigration? Documentos de Trabajo N. 1503. Madrid: Banco de España. 
Jeanpierre, B., \& Hallett-Njuguna. R. (2014). Exploring the science attitudes of urban diverse gifted middle school students, Creative Education, 5(16), 1492-1496. doi: 10.4236/ce.2014.516166 Kali, Y \& Linn, C. (2008). Designing Effective Visualizations for Elementary School Science. The elementary school journal, 109 (2) 181-198

Korpershock H., Kuyper. H. \& Wert. G van der. (2015). Differences in students' school motivation: A latent class modelling approach, Social Psychology of Education, 18,137-163. doi: 10.1007/s11218014-9274-6

Long, D. (2013). Science Education in a Secular Age, Cultural Studies of Science Education, 8(1), 25-38. doi:10.1007/s11422-012-9434-y Lloyd, G.E.R. (2009). Disciplines in the Making. Cross-Cultural

Perspectives on Elites, Learning, and Innovation. Oxford. Oxford University Press.

MECD (2016). Datos y cifras del sistema universitario español. Curso 2015/2016. Madrid: Subdirección General de Documentación y Publicaciones.

MECD (2015). Datos y cifras del sistema universitario español. Curso 2014/2015. Madrid: Subdirección General de Documentación y Publicaciones.

Montoro Sánchez, M. A., \& Mora Valentín. E.M, (2006). Hacia una gestión eficaz de las relaciones entre empresas y universidades, Universia Business Review, 010, 38-53.

Murphy, C., \& Beggs. J. (2003). Children's perceptions of school science, School Science Review, 84(308), 1009-116.

National Science Board (2003) Broadening Participation in Science and Engineering Research and Education. Virginia: National Science Foundation.

National Science Foundation (2014) Science and Engineering Indicators 2014. Virginia: NSF.

OCDE. (2012). Informe Pisa. Programa para la evaluación internacional de los alumnos. Madrid: MECD.

Pérez de Landazábal, C. (coord.) (2012). La relevancia social de la educación científica. Madrid: UNED.

Polino, C. (comp.) (2011). Los estudiantes y la ciencia: encuesta a jóvenes iberoamericanos, Buenos Aires: OEI 
Popper, K. R. (2000). En busca de un mundo mejor. Barcelona: Paidos. Porto Castro, A-M., \& Mosteiro García. M-J, (2000). Los motivos de elección de estudios en alumnos y alumnas de universidad, Innovación educativa, 10, 121-132.

Porter, A.L (et al.) (2006). Interdisciplinary research: meaning, metrics and nurture. Research Evaluation, 15, (3), 187195. doi.org/10.3152/147154406781775841

Quaglatia, A.B. (2015). University Festival Promotes STEM Education, Journal of STEM education, 16(3), 20-23.

Randstad (2015) Randstad Employer Branding: cuando la percepción puede convertirse en realidad. Madrid: Randstad

Rocard, M. (coord.) (2007). Science Education Now: A Renewed Pedagogy for the Future of Europe. Bruselas: European Commision.

Rodríguez Pomeda, J., \& Casani Fernández de Navarrete. F. (2007). La transferencia de tecnología en España. diagnóstico y perspectivas, Economía industrial, 366, 15-22.

Rundell, S. (2013), "STEM Education: Time for Integration". Peer Review, $13,(3), 1-8$

Sanabria Landazabal, N. (2011). Investigación y desarrollo (I+D) en la productividad, Dimensión empresarial, 9, 55-63.

Schwab, K. (ed) (2014). The Global Competitiveness Report 2014-2015. Geneve: World Economic Forum.

Sjøberg. S., y C, Schreiner. (2010). The ROSE Project. An overview and key findings. Recuperado de:

http://roseproject.no/network/countries/norway/eng/nor-SjobergSchreiner-overview-2010.pdf.

Shiel. G., \& Cartwright. F. (2015), Analyzing Data from a National Assessment of Educational Achievement. Washington DC: World Bank.

Strayhorn, T.S. (2010). The Role of Schools, Families, and Psychological Variables on Math Achievement of Black High School Students. The High School Journal, 93(4), 177-194.

Suchman, E.L. (2014). Changing academic culture to improve undergraduate STEM education, Trends in Microbiology, 22, (12), 657-659.

Svensmark, H., \& Calder. N. (2007). The Chilling Stars: A New Theory of Climate Change. London: Icon Books. 
UNESCO (2014) UNESCO Science: for Peace and Sustainable Development. Paris: UNESCO

Valero-Matas, J.A., Valero-Oteo, I., Coca, R. J., \& Laurencio Leyva, A. (2016). Creatividad y educación para el siglo XXI desde una perspectiva sociológica. RIPS: Revista de Investigaciones Políticas y Sociológicas, 15(2). doi:10.15304/rips.15.2.3473

Van Zee, E.H. (et al). (2005) Learning and teaching science as inquiry: A case study of elementary school teachers' investigations of light.

Science Education, 89, 1007-1042. DOI.10.1002/sce.20084

Vázquez-Alonso, Á., \& Manassero-Mas. M-A. (2015). La elección de estudios superiores científico-técnicos: análisis de algunos factores determinantes en seis países, Revista Eureka sobre Enseñanza y Divulgación de las Ciencias, 12(2), 264-277.

Vázquez-Alonso, Á., \& Manassero-Mas. M-A. (2012). La selección de contenidos para enseñar naturaleza de la ciencia y tecnología (parte 1): Una revisión de las aportaciones de la investigación didáctica, Revista Eureka sobre Enseñanza y Divulgación de las Ciencias, 9(1), 2-31.

Vázquez-Alonso, Á., \& Manassero-Mas. M-A. (2008). El declive de las actitudes hacia la ciencia de los estudiantes: un indicador inquietante para la educación científica, Revista Eureka sobre Enseñanza y Divulgación de las Ciencias, 5(3), 274-292

Vázquez-Alonso, Á., \& Manassero-Mas. M-A. (2005). La ciencia escolar vista por los escolares, Bordón, 57(5), 717-736.

Zembal-Saul, C. (2009). Learning to teach elementary school science as argument. Science Education, 93, (4) 687-719.

Zuñiga, P. (2010). La necesidad de innovar: el camino hacia el progreso de América latina y el Caribe. Washington DC: Banco Interamericano de Desarrollo.

Jesús Alberto Valero-Matas is professor at the University of Valladolid, Spain

Irene Valero-Oteo is PhD student at the University of País Vasco, Spain

Juan Romay Coca is professor at the University of Valladolid, Spain

Contact Address: javm2011@icloud.com 